\title{
AN ENHANCED DIFFERENCE PAIR MAPPING STEGANOGRAPHY METHOD TO IMPROVE EMBEDDING CAPACITY
}

\author{
V.Poongavanam ${ }^{1}$, L. Mary Shamala ${ }^{2}$ \\ ${ }^{I}$ Master of Engineering, Computer Science and Engineering, IFET College of Engineering, Tamilnadu, India \\ ${ }^{2}$ Associate Professor, Computer Science and Engineering, IFET College of Engineering, Tamilnadu, India
}

\begin{abstract}
Reversible Data Hiding $(R D H)$ aims to embed secret message into a cover image by slightly modifying its pixel values, and unlike data hiding, the embedded message as well as the cover image should be completely recovered from the marked content. Many methods have been implemented in RDH to maintain secure confidential data, such as Difference Expansion (DE), Histogram Modification and Difference Pair Method (DPM). But its embedding capacity is quite low and also its PSNR value is low, then it takes more time to complete the process. In order to overcome these issues, Modified Difference Pair Mapping (MDPM) technique of RDH is proposed for embedding extra information in an image by making small modifications to its pixels. MDPM is the process of dividing an image into blocks. Then divide the blocks into embeddable area and non-embeddable area. Embeddable area is used to embed a secret message, and non-embeddable area is used to record the side information. In embeddable area, Centre pixel of $3 \times 3$ blocks is chooses as reference pixel, remaining 8 pixels is referred to as non-reference pixels. Then calculate difference value in nonreference pixels. Finally difference histogram has been generated. In that difference histogram, peak point and zero point is obtained. From this peak point and zero point of difference histogram, secret message is embedding. Location map and side information is used in non-embeddable area to extract a secret message and original image. Theoretical analysis indicates that the proposed technique can provide good trade-off between embedding capacity and stego-image quality.
\end{abstract}

Keywords: Difference Value calculation, Local complexity, Reversible Data Hiding, Reference and Non-reference pixels, Histogram generation, Histogram Pair, Smooth region and Complex region.

\section{INTRODUCTION}

The hiding of data is frequently called steganography. Steganography is a technology that hides a message within an object. In that steganography, Reversible Data Hiding (RDH) is the one of the scheme to hide a secret data. It is also same as like data hiding, but unlike data hiding, RDH can recover the embedded message as well as original image without any distortion. Nowadays, RDH can be preferably used to hiding a secret message. Generally, the performance of a RDH scheme is evaluated by the capacity-distortion behavior. For achieving a high embedding capacity (EC) and to obtain a good marked image quality we have to reduce the embedding distortion as much as possible. RDH Scheme has been used to hide secret data into an image. Many approaches have been implemented in RDH to provide secure data such as difference expansion, histogram modification and Difference pair. The first histogram-based RDH method is the Histogram Shifting [1]. This method uses peak and minimum points of the pixelintensity-histogram to embed data. It changes each pixel value at most by 1 , and thus a good marked image quality can be obtained. However, its EC is quite low and this method does not work well if the cover image has a flat histogram. To facilitate it, Lee et al. [2] proposed to utilize the differencehistogram instead. This method exploits the correlation among neighboring pixels and can embed larger payload with reduced distortion compared with $\mathrm{Ni}$ et al.'s. Lee et al.'s method can be in fact implemented, in an equivalent way, by modifying the two-dimensional pixel-intensity-histogram according to a pixel-pair-mapping (PPM) which is an injective mapping defined on pixel-pair.

In Difference Pair Mapping method, LSB algorithm is used for embedding the data. But these methods are consuming more time to complete the process, also PSNR value is low. This paper presents a new method Modified Difference pair mapping (MDPM) based on RDH scheme to achieve a high embedding capacity and better PSNR value.

We remark that, RDH methods generally contain two basic steps:

- Histogram generation: First, each local image region consisting of several pixels i.e. pixel-pair is projected to a onedimensional space to get a difference value of pixel-pair. Onedimensional histogram (e.g., difference- histogram) is generated by counting the number of difference pair value.

- Histogram modification: Finally, embed data into the cover image by modifying the histogram. In most cases, the histogram bins with high frequencies are expanded to carry data while some others are shifted to ensure the reversibility. 
By using the two-dimensional difference-histogram and this specific DPM, compared with the conventional histogram based methods, more pixels are used for carrying data while the number of shifted pixels is reduced as well, and thus an improved embedding performance is achieved.

The rest of the paper is organized as follows. The related works are briefly introduced in Section II. Section III presents the proposed RDH scheme in details. The comparisons with the prior works are shown in Section IV. Section V concludes this paper.

\section{RELATED WORKS}

Difference Expansion is the process of dividing the image into pair of pixels and a secret message was embedded into the difference between the pixels. DE first selects the adjacent pixels, and then expands the difference values of two pixels to embed the secret message. The secret message was embedded using a location map which contains the location of data carrying pixels. Using this difference expansion method can achieve sufficient embedding capacity. But Difference Expansion method suffers from low compressibility of location image and lack of embedding capacity control. Location map is utilized to record the pixels that may cause the overflow problem. This method provides less embedding capacity and allows limited bits of data to embed.

To overcome this issue, RDH introduced Histogram modification method to embed a data into an image based on pixel pair method. Histogram Modification is a process of scanning the image and selecting the pixel pair, and then calculating a difference value to form histogram. Then find a peak point in that difference value to embed a data. This method is used to provide lossless large volume data hiding method. Embedding and extraction process is based on the difference and pixel value.

Difference value for pixel-pair:

$$
\left\{\begin{array}{l}
\{x i \quad \text {,if } \mathrm{i}=0 \\
\{x i-1-x i \mid, \text { otherwise } 0
\end{array}\right.
$$

Then have to select peak point in those pixel pair value. In that peak point, if di>P, shift each pixel by 1 unit. Then,

$\mathrm{Yi}=\left\{\begin{array}{c}x_{i}, \quad \text { if } i=0 \\ x_{i+1}, \quad \text { if } d i>P, x i>x i-1 \\ x_{i-1}, \quad \text { if } d i>P, x i<x i-1\end{array}\right.$

Yi= embedded value of pixel $\mathrm{i}$.

$\mathrm{d} i=\mathrm{P}$ modify the pixel $\mathrm{xi}$, according to the message bit $\mathrm{b}$.

$$
\begin{aligned}
& \left\{x_{i+b} \text { if } \mathrm{x}_{\mathrm{i}}>\mathrm{x}_{\mathrm{i}-1}\right. \\
& \left\{x_{i-b} \text { if } \mathrm{x}_{\mathrm{i}}<\mathrm{x}_{\mathrm{i}-1}\right.
\end{aligned}
$$

Based on these difference value and peak point value embedding has to be done here. If difference value and peak point value should be equal means, it should modify the pixel value by message bit $b$.

\subsection{Embedding Process}

Step 1: First, it scans the image.

Step 2: Calculate the pixel difference (di), between adjacent pixels $(\mathrm{Xi}, \mathrm{Xi}-1)$ and generate the histogram.

Step 3: Determine the peak point $(\mathrm{P})$, from pixel differences.

Step 4: If the di> $\mathrm{P}$ means, it shifts each pixel by 1 unit.

Step 5: If $\mathrm{di}=\mathrm{P}$ means, it modify the pixel $\mathrm{Xi}$ according to message bit $b$, by embedding this message bit into pixel value.

\subsection{Extraction Process}

Step 1: Extraction is the reverse process of this embedding process, then it restores the pixel value to extract a secret message from the cover image without any distortion.

Step 2: Scan the image in the same order as in the embedding phase.

Step 3: Using the difference pixel $\left(\mathrm{d}_{\mathrm{i}}\right)$ values and peak point (P) values it restores the pixel in the stego image to extract a secret data.

Step 4: Extract a secret data from cover image without any distortion using pixel restoration.

This method provides better embedding capacity, But this method will not work, if the difference value produces flat histogram.

To overcome this issue, Difference Pair Mapping has been proposed in RDH method. DPM is the process of dividing an image into a pixel, and then it selects an adjacent pixel to form a pixel-pair. Using this pixel pair by considering a pixel-pair and its context, a local image region is projected to a twodimensional space to obtain a sequence consisting of difference-pairs. Then, a two-dimensional differencehistogram is generated by counting the difference-pairs. Finally, reversible data embedding is implemented according to a specifically designed difference-pair-mapping (DPM). DPM is a natural extension of expansion embedding and shifting techniques used in current histogram-based methods. By using the two-dimensional difference-histogram and this specific DPM, compared with the conventional histogram based methods, more pixels are used for carrying data while the number of shifted pixels is reduced as well, and thus an improved embedding performance is achieved. 
For a pixel-pair(x, y), to compute difference values $d 1=$ $x-y$ and $d 2=y-z$ to form a two-dimensional difference-histogram of, where $\mathrm{z}$ is a prediction of $\mathrm{y}$.

In this situation, since pixel-pair $(\mathrm{x}, \mathrm{y})$ has four modification directions, the difference-pair $\left(\mathrm{d}_{1}, \mathrm{~d}_{2}\right)$ also has four modification directions.

For example, by modifying y to $y+1$, the modification direction to $(\mathrm{x}, \mathrm{y})$ is "up" and the corresponding modification direction to $\left(d_{1}, d_{2}\right)$ is "upper-left", since $d_{1}$ changes to $d_{1}-1$ and $d_{2}$ changes to $d_{2}+1$. Based on these four modification directions, DPM have been implemented. But in this DPM method based RDH, only one pixel of a pixel-pair should be modified by one bit. So this method provides low embedding capacity.

Our method is motivated by the aforementioned works and will be described in details in next section.

\section{PROPOSED WORK}

RDH scheme introduces DPM method to embed a data into an image. This Section is organized as follows. First in Section 3.1 Modified DPM-based scheme. In this way, by extending difference- histogram (or, in an equivalent way, twodimensional pixel-intensity-histogram) to two-dimensional difference-histogram, the proposed scheme extends current histogram-based RDH methods. The detailed embedding and extraction procedures of this scheme are summarized in Section3.2 and section 3.3. Finally, Experimental results are explained in Section 3.4 .

\subsection{Modified DPM based Scheme}

MDPM is the process of dividing an image into embeddable area and non-embeddable area. Fig.1 explains the overall process of MDPM. The embeddable area is used for embedding secret data and the non-embeddable area is used to record the information of location map and side information. To embed a secret data, first divide an embeddable area into $3 \times 3$ blocks. For each block, the center pixel is selected as the reference pixel, and the other eight pixels are referred to as non-reference pixels.

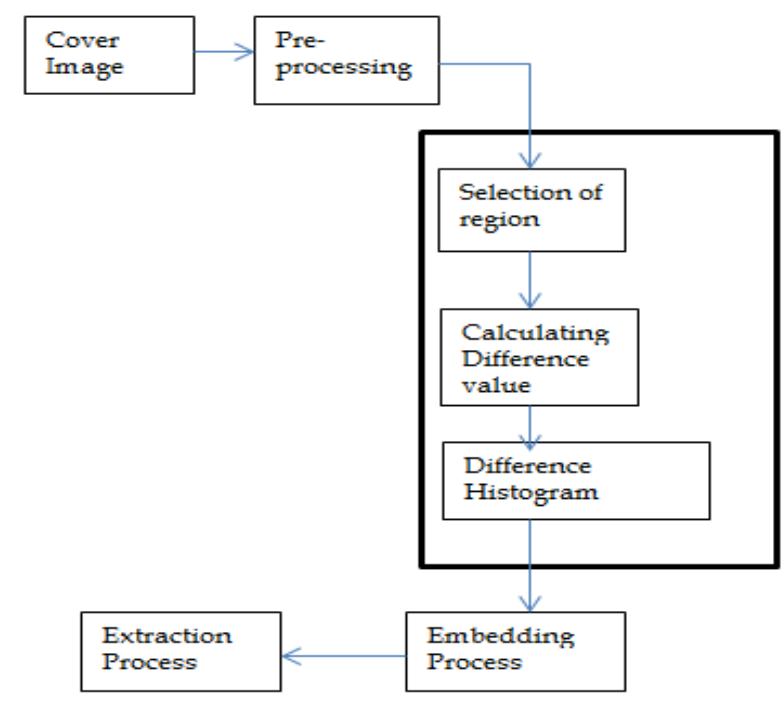

Fig.1. Architecture diagram

Then calculate a difference value for those non-reference pixels. Using this difference value of non-reference pixels histogram is generated. From that histogram, pairs of zero point and peak point is obtained. Using this pair, secret data is embedded into difference value. Finally, reversible data embedding is implemented according to a specifically designed MDPM. Then to extract a secret message from original image location map has been used.

\subsubsection{Local Complexity and Selection of Image Region}

The Cover image I is divided into block with the size of $3 \times 3$ pixels. Then select the embeddable area and non-embeddable area using the measurement of the local complexity.

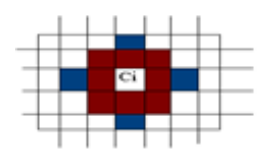

Satellite reference pixels

Non-Reference pixels

Fig.2. Image block with reference pixel $C_{i}$, satellite reference pixel and non-reference pixels.

Centre pixel $C_{i}$ and $C^{u}, C^{d}, C^{r}, C^{1}$ and 1, 2, 3, 4, 5, 6, 7, 8 mentioned in Fig.2 reffered as reference pixel, satellite reference pixels and non-reference pixels respectively. Local Complexity is measured by following equation (3.1) 


$$
L C_{i}=\text { Range }\left(\mathrm{C}_{\mathrm{i}}{ }^{1}, \mathrm{C}_{\mathrm{i}}^{\mathrm{r}}, \mathrm{C}_{\mathrm{i}}^{\mathrm{u}}, \mathrm{C}_{\mathrm{i}}^{\mathrm{d}}\right)
$$

Where the Range $\left(\mathrm{C}_{i}^{1}, \mathrm{C}_{\mathrm{i}}^{\mathrm{r}}, \mathrm{C}_{\mathrm{i}}^{\mathrm{u}}, \mathrm{C}_{\mathrm{i}}^{\mathrm{d}}\right)$ function is used to measure the maximum and minimum values of four satellite reference pixels $\mathrm{C}_{\mathrm{i}}^{1}, \mathrm{C}_{\mathrm{i}}^{\mathrm{r}}, \mathrm{C}_{\mathrm{i}}^{\mathrm{u}}, \mathrm{C}_{\mathrm{i}}^{\mathrm{d}}$. If the complexity value is less than the threshold value $\mathrm{T}$, the current processing block is located in smooth region, otherwise the block is located in complex region. Smooth region is used here to embed secret data. Complex region is used here to carrying location map and side information.

\subsubsection{Calculating Difference Value and Histogram}

\section{Generation}

Smooth block is used to embedding a secret data, compared to complexity region smooth block provide more embedding capacity. Fig 3.a and b shows the process of pixel block and difference block. Then smooth block non reference pixel is used to embed a data. First have to select reference and nonreference pixels in smooth block. Then have to calculate difference value for those non-reference pixels.

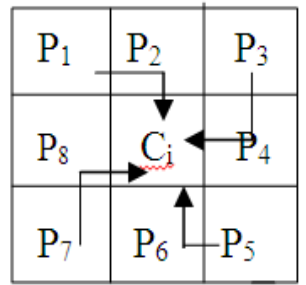

Fig.3.a. Pixel blocks

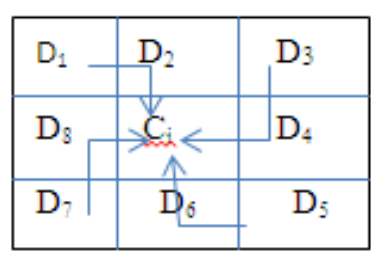

Fig.3.b. Difference block
The difference histogram of the non-reference pixels is constructed. Then, two pairs $\left(\mathrm{PP}_{1}, \mathrm{ZP}_{1}\right)$ and $\left(\mathrm{PP}_{2}, \mathrm{ZP} 2\right)$ of the peak and zero points of the histogram are obtained. Based on these pairs, embedding should be processed in difference block.

\subsection{Data Embedding Phase}

During the embedding process, the histogram shifting technique is used in the proposed scheme. Fig.4 shows the embedding process. Based on the difference value of nonreference pixels histogram have been generated. From that histogram pair (peak point and zero point) value embedding should be processed here.

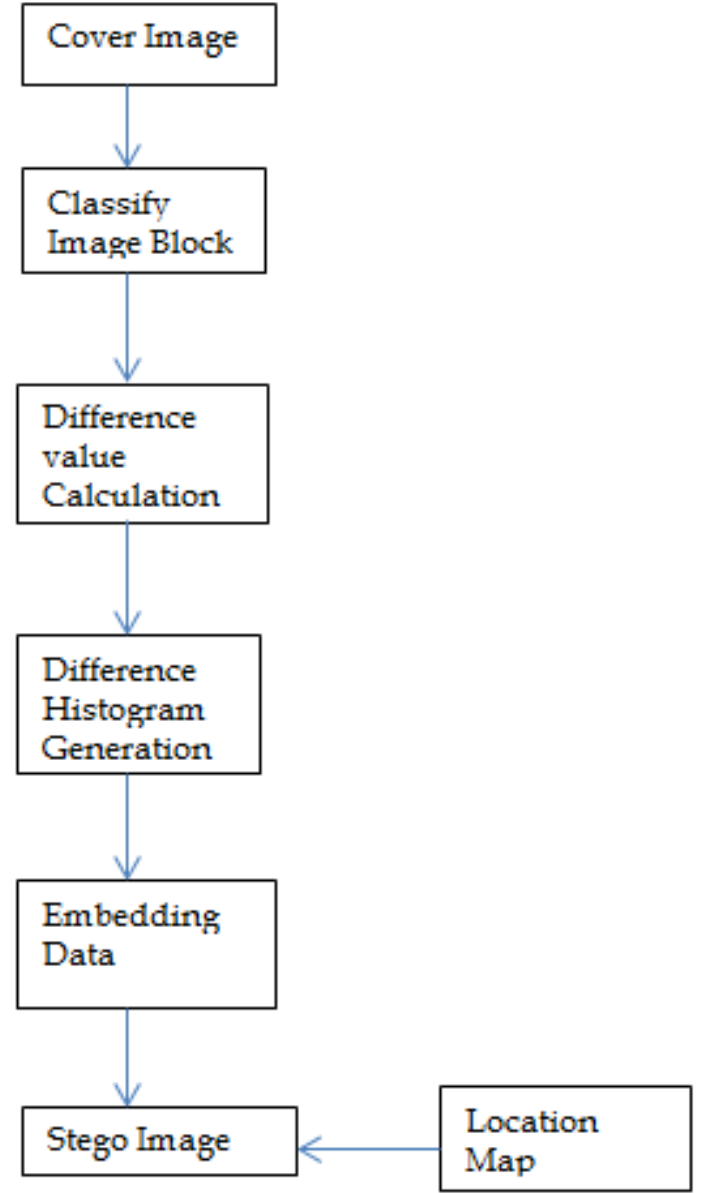

Fig.4 Embedding Process

The embedding process should be explained in the following steps:

Step 1: The cover image I is partitioned into two areas, i.e., embeddable area and non-embeddable area.

Step 2: The embeddable area of the cover image is divided into blocks with sizes of $3 \times 3$ pixels.

Step 3: For each block $\mathrm{B}_{\mathrm{i} \epsilon}$ S-block, compute the difference value $\mathrm{d}_{\mathrm{i}}$ of the non-reference pixels in $B_{i}$ with equation-1

$$
\begin{aligned}
& \mathrm{d}_{1}=\mathrm{P}_{1}-\mathrm{P}_{2} \\
& \mathrm{~d}_{2}=\mathrm{P}_{2}-\mathrm{C}_{\mathrm{i}} \\
& \mathrm{d}_{3}=\mathrm{P}_{3}-\mathrm{P}_{4} \\
& \mathrm{~d}_{4}=\mathrm{P}_{4}-\mathrm{C}_{\mathrm{i}} \\
& \mathrm{d}_{5}=\mathrm{P}_{5}-\mathrm{P}_{6} \\
& \mathrm{~d}_{6}=\mathrm{P}_{6}-\mathrm{C}_{\mathrm{i}} \\
& \mathrm{d}_{7}=\mathrm{P}_{7}-\mathrm{P}_{8} \\
& \mathrm{~d}_{8}=\mathrm{P}_{8}-\mathrm{C}_{\mathrm{i}}
\end{aligned}
$$

Step 4: The difference histogram of the non-reference pixels is constructed. Then two pairs, i.e., $\left(\mathrm{PP}_{1}, \mathrm{ZP}_{1}\right)$ and $\left(\mathrm{PP}_{2}, \mathrm{ZP}_{2}\right)$ of the peak and zero points of the histogram are obtained. 
Step5: Scan the difference values di sequentially. If $\mathrm{d}_{\mathrm{i}}=\mathrm{PP} 1$ or $\mathrm{d}_{\mathrm{i}}=\mathrm{PP} 2$, a secret bit can be embedded. Otherwise no difference secret bit can be embedded.

$$
d i^{\prime}=\left\{\begin{array}{c}
d_{i} \quad \text { if } S=0 \\
d_{i-1} \text { if } s=1 \text { and } d_{i}=P P 1 \\
d_{i+1} \quad \text { if } S=1 \text { and }_{i}=P P 2
\end{array}\right.
$$

Step6: Repeat step 5 until the embedded data S are embedded completely.

These are steps to complete the embedding process, after finishing these steps stego image is obtain. Then extraction process will be continued to recover the cover image as well as secret message.

\subsection{Data Extraction Phase}

In the extraction phase, the extra information, including location map and side information should be reconstructed. Non-embeddable area is used here to record the information of location map and side information. To reconstruct he cover image, the LSBs of the pixels in the non-embeddable area are recovered.

The extraction phase should be explained in the following steps:

Step 1: The stego image I" is divided into two areas, i.e., the embeddable area and the non-embeddable area, in the same manner used in the embedding phase.

Step 2: Divide the embeddable area of the stego image into blocks with sizes of $3 \times 3$ pixels.

Step3: Compute the difference values of the non-reference pixels in $\mathrm{B}^{\prime} \mathrm{i}$ using equation 1.

Step4: Scan the difference values d'i sequentially by using the same order that was used in the data embedding phase.

Step5: The original difference value $\mathrm{d}_{\mathrm{i}}$ can be reconstructed using equation 3

$d i=\left\{\begin{array}{cl}d^{\prime}{ }_{i+1} & \text { if } Z P 1<d i<P P 1 \\ d^{\prime}{ }_{i-1} & \text { if } P P 2<d i<Z P 2 \\ & d^{\prime}{ }_{i} \text { otherwise }\end{array}\right.$

Step 6: Repeat step 5 until all embedded data $\mathrm{S}$ have been extracted.

After finishing these steps stego image and secret message is recovered. To reconstruct the cover image $I$, the LSBs of the pixels in the non-embeddable area are recovered according to the embedded data $S$.

\subsection{Experimental Results}

DPM based on LSB algorithm has been given below in table1, based on these results proposed work have been improve to overcome these issues in their work.
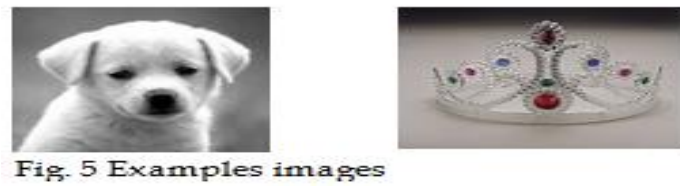

Based on these results proposed work have been implemented. Fig. 5 shows the example image to calculate the embedding capacity.

Table.1. Embedding Capacity

\begin{tabular}{|l|l|l|l|}
\hline S.no & $\begin{array}{l}\text { DE (Embedding } \\
\text { Capacity in \%) }\end{array}$ & $\begin{array}{l}\text { Histogram } \\
\text { modification } \\
\text { (Embedding } \\
\text { Capacity \%) }\end{array}$ & $\begin{array}{l}\text { DPM using } \\
\text { LSB } \\
\text { (Embedding } \\
\text { Capacity in } \\
\%)\end{array}$ \\
\hline 1. & 39.4444 & 40.123 & 41.1111 \\
\hline 2. & 30.1133 & 32.2233 & 33.27160 \\
\hline
\end{tabular}

MDPM method is used to provide high embedding capacity and high PSNR value.

\section{CONCLUSIONS}

Reversible Data Hiding is a process of embedding a secret data into an image, which allow exact recovery of the original image by extracting secret message from the marked image without any distortion. Existing RDH methods like Difference Expansion, Histogram Modification and Difference Pair Mapping method provides less image quality. In order to solve this issue, a better method is proposed in this project to improve the image quality. Theoretical analysis reveals that MDPM method can exploit the better image redundancy and achieve sufficient embedding capacity.

\section{ACKNOWLEDGMENTS}

The authors wish to thank Principal Dr.G.Mahendiran, Dean Academics Professor S.Matilda and Associate Professor L.Mary Shamala for giving the valuable suggestion and support. 


\section{REFERENCES}

[1] Weiming Zhang, Xinlu Gui, Xiao long Li, , and Bin Yang, "Reversible data hiding by two dimensional difference histogram", IEEE Transactions On Information Forensics And Security, Vol. 8, NO. 7, JULY 2013.

[2] C. C. Chang, W. L. Tai, C. M. Yeh, and, "Reversible data hiding based on histogram modification of pixel differences," IEEE Trans. Circuits Syst. Video Technol., vol. 19, no. 6, pp. 906-910, Jun. 2009.

[3] V. Fotopoulos ,E. Varsaki, , A. N. Skodras, "A reversible data hiding technique" in Proc Technical Report HOU-CS-TR-2006-08-GR. A. M.

[4] Alattar, "Reversible watermark using the difference expansion of a generalized integer transform," IEEE Trans. Image Process., vol. 13, no. 8, pp. 1147-1156, Aug. 2011.

[5] Y. Hu, H. K. Lee, and J. Li, "DE-based reversible data hiding with improved overflow location map," IEEE Trans. Circuits Syst. Video Technol., vol. 19, no. 2, pp. 250-260, Feb. 2009.

[6] Nag ham Hamid, Abid Yahya, R. Badlishah Ahmad \& Osamah M. Al-Qershi, "Image Steganography Techniques: An Overview", International Journal of Computer Science and Security (IJCSS), Volume (6) : Issue (3) : 2012.

[7] Shashikala Channalli, Ajay Jadhav,"Steganography An Art of Hiding Data" International Journal on Computer Science and Engineering Vol.1(3), ISSN : 0975-3397 2009.

[8] Hsien-Wei Yang1,2, I-En Liao And Chaur-Chin Chen, "Reversible Data Hiding Based on Median Difference Histogram", International journal of information science and engineering 27, 577-593 (2011).

[9] Yun Q. Shi "Statistical Modeling in Reversible Data Hiding", International Journal of Computer Science and Security (IJCSS), 2011.

[10] Ching-Yu Yang \& Wu-Chih Hu, "Reversible Data Hiding in the Spatial and Frequency Domains", International Journal of Image Processing, Volume (3):Issue (6), 2011.

[11] A. E.Mustafa A.M.F.ElGamal M.E.ElAlmi Ahmed.BD, "A Proposed Algorithm for Steganography in Digital Image Based on Least Significant Bit", Research Journal Specific Education, Issue No. 21, April. 2011.

[12] Chang-Chun Chan, Chin-Chan Chang," Hiding Secret Information Lossless Compression codes", International Journal of Innovative Computing, vol.7, no.10, October 2011, ISSN 1349-4198.

[13] Yun Q. Shi1, Zhicheng Ni, Dekun Zou, Changyin Liang and Guorong Xuan, "Lossless data hiding: Fundamentals, algorithms and applications", ISCAS, vol.4, 2004.
[14] Hyoung Joong Kim, Vasiliy Sachnev, and Dong Hoi Kim, "New reversible data hiding algorithm based on difference expansion method", vol.7, no.5 2005.

[15] Vinit Agham and Tareek Pattewar1, "A survey on Seperable Reversible Data hiding", IMACST: Volume 4 Number 1 May 2013 1857-7202.

\section{BIOGRAPHIES}

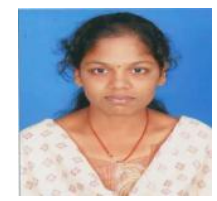

Poongavanam.V(1991) received the degree B.Tech,IT from Anna University, Chennai in 2012. She currently pursues Master of Engineering, in Computer Science under the same University. Her research interests include Steganography, Image Processing and Data Mining.

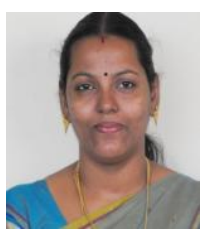

Mary Shamala.L has pursued M.Tech degree with specialization in Information security from Pondicherry Engineering College, Puducherry, India. She is currently an Associate Professor in IFET College of Engineering, Tamilnadu, India. Her research interests include network and information security, privacy enhancing technologies in social computing and lightweight cryptography. 